\title{
Interfacial instability in finite elasticity under initial stress
}

\author{
By M. A. Biот
}

Shell Development Company (A Division of Shell Oil Company), Exploration and Production Research, Division, Houston, Texas

\section{(Communicated by Sir Edward Bullard, F.R.S._Received 12 September 1962)}

\begin{abstract}
It is shown that two homogeneous elastic media of different rigidities adhering at a plane interface will become unstable at the surface of discontinuity when a certain critical magnitude of initial stress is attained. The general characteristic equation for the interfacial instability is derived for a general case of elastic anisotropy. The critical stress is evaluated as a function of the rigidity ratio of the two media in the particular case of isotropy.
\end{abstract}

\section{INTRODUCTTON}

In a previous paper $(1963 b)$ the author has investigated the surface instability of an elastic anisotropic half-space under initial stress in finite strain. Our purpose here is to extend the analysis to the stability at the plane interface of two adhering anisotropic half-spaces. Each half-space is homogeneous, and its elastic properties are discontinuous at the interface. The two media are orthotropic with parallel axes of symmetry, and one of the planes of symmetry lies parallel with the interface.

We consider a state of finite initial strain with its principal directions along the axes of elastic symmetry. Hence, the principal initial stresses are also directed along the same axes.

The case of isotropy for one or both of the two media is, of course, included as a particular case, since any anisotropy induced by the initial stress will not disturb the axes of symmetry.

The author has pointed out in several earlier papers that the dynamic analogue of surface instability is the Rayleigh wave. In the same way the analogue of the interfacial instability is the Stoneley wave.

We are interested here in finding spontaneous deformation at the interface due to instability which causes the disturbance to vanish exponentially with the distance from the discontinuity. From the previous analysis $(1963 b)$ of the free surface the exponential may or may not contain an oscillatory factor.

For the instability to be confined to the interface, the initial stress and the elastic parameters must be such that internal buckling does not occur. We shall assume that this is the case. The criteria for this requirement have been discussed in a previous paper (1963a).

\section{General equations}

Two half-space incompressible elastic media of orthotropic or isotropic properties are adhering at a plane interface. The interface and the directions of elastic symmetry are parallel with the co-ordinate axes. The $x$ axis is located at the interface (figure 1), and the $y$ axis is perpendicular to it. The medium is assumed to be in an 
initial state of finite strain with principal directions along the axes of elastic symmetry. In the $x$ direction, initial compressions $P$ and $P^{\prime}$ are assumed to be produced.

We shall consider the stability of the interface for an incremental plane strain parallel with the $x, y$ plane. The equations are independent of any component of

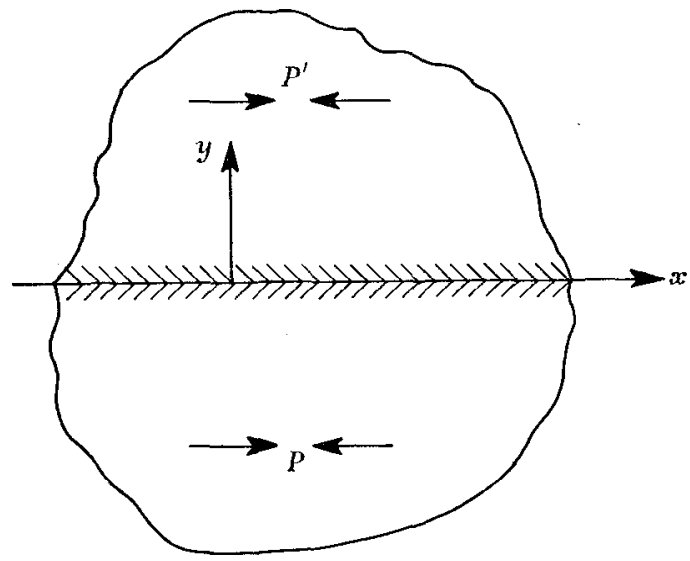

Fraure 1. Two semi-infinite elastic media with adhering interface at $y=0$.
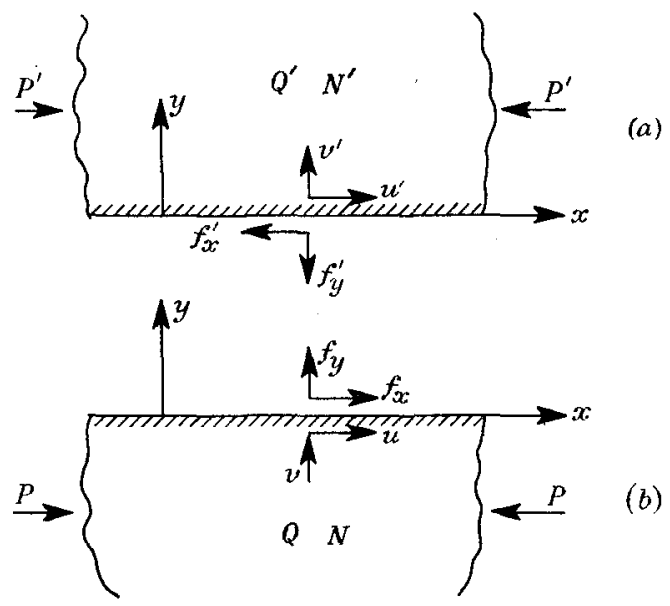

Fiaure 2. Forces and displacements at the interface.

initial stress perpendicular to the $x, y$ plane. Moreover, the results of the present analysis are also valid for the case of a triaxial state of initial stress, provided $P$ and $P^{\prime}$ are replaced by the stress differences in the $x, y$ plane. Hence it is sufficient to analyze the case of uni-axial initial compression.

Let us separate the top and bottom media as indicated in figure 2. A sinusoidal deformation of the interface produces forces of components $f_{x}$ and $f_{y}$ on the surface of the bottom medium (figure $2 b$ ):

$$
\left.\begin{array}{l}
f_{x}=\tau \sin l x \\
f_{y}=q \cos l x
\end{array}\right\}
$$


These components represent the force acting on an element of the surface of unit area before the incremental deformation is applied. The surface displacements are

$$
\left.\begin{array}{l}
u=U \sin l x \\
v=V \cos l x
\end{array}\right\}
$$

The relation between the surface forces and displacements has been evaluated in a previous paper $(1963 b)$. The relation is

$$
\left.\begin{array}{l}
\tau / l L=a_{11} U+a_{12} V \\
q / l L=a_{12} U+a_{22} V
\end{array}\right\}
$$

The coefficients are

$$
\left.\begin{array}{l}
a_{11}=\sqrt{ }\{2(m+k)\}, \\
a_{12}=k-1, \\
a_{22}=k \sqrt{ }\{2(m+k)\},
\end{array}\right\}
$$

with

$$
\left.\begin{array}{r}
k=\sqrt{\frac{1-\zeta}{1+\zeta},} \quad m=\frac{1}{1+\zeta}\left(\frac{2 N}{Q}-1\right), \\
\zeta=P / 2 Q, \quad L=Q+\frac{1}{2} P .
\end{array}\right\}
$$

In these expressions the quantities $N$ and $Q$ are the incremental elastic moduli of the bottom medium for plane incremental strain. They correspond to the stressstrain relations

$$
\left.\begin{array}{rl}
s_{11}-s & =2 N e_{x x} \\
s_{22}-s & =2 N e_{y y} \\
s_{12} & =2 Q e_{x y}
\end{array}\right\}
$$

which were explained in detail earlier $(1963 a, b)$.

Similarly, we may consider forces acting on the surface of the upper medium (figure $2 a$ ). The components of these forces along the negative directions of the axes are written

$$
\left.\begin{array}{l}
f_{x}^{\prime}=\tau^{\prime} \sin l x, \\
f_{y}^{\prime}=q^{\prime} \cos l x .
\end{array}\right\}
$$

The corresponding displacements are

$$
\left.\begin{array}{rl}
u^{\prime} & =U^{\prime} \sin l x \\
v^{\prime} & =V^{\prime} \cos l x
\end{array}\right\}
$$

Relations between forces and deformation may be derived from equations $(2 \cdot 3)$ for the bottom medium after appropriate changes in sign. We find

$$
\left.\begin{array}{l}
\tau^{\prime} \mid l L^{\prime}=-a_{11}^{\prime} U^{\prime}+a_{12}^{\prime} V^{\prime} \\
q^{\prime} \mid l L^{\prime}=a_{12}^{\prime} U^{\prime}-a_{22}^{\prime} V^{\prime}
\end{array}\right\}
$$

The coefficients are defined by expressions similar to $(2 \cdot 4)$ and $(2 \cdot 5)$ with primes to indicate that they refer to the parameters of the upper medium.

$$
\left.\begin{array}{l}
a_{11}^{\prime}=\sqrt{ }\left\{2\left(m^{\prime}+k^{\prime}\right)\right\} \\
a_{12}^{\prime}=k^{\prime}-1 \\
a_{22}^{\prime}=k^{\prime} \sqrt{ }\left\{2\left(m^{\prime}+k^{\prime}\right)\right\}
\end{array}\right\}
$$


with

$$
\left.\begin{array}{r}
k^{\prime}=\sqrt{\frac{1-\zeta^{\prime}}{1+\zeta^{\prime}}, \quad m=\frac{1}{1+\zeta^{\prime}}\left(\frac{2 N^{\prime}}{Q^{\prime}}-1\right),} \\
\zeta^{\prime}=P^{\prime} / 2 Q^{\prime}, \quad L^{\prime}=Q^{\prime}+\frac{1}{2} P^{\prime} .
\end{array}\right\}
$$

At the interface the stresses and displacements must be the same. Therefore, we must write

$$
\tau=\tau^{\prime}, \quad q=q^{\prime}, \quad U=U^{\prime}, \quad V=V^{\prime} .
$$

\section{Discusston OF INTERFACIAL STABILITY}

Substituting the values $(2 \cdot 3)$ and $(2 \cdot 9)$ into equations $(2 \cdot 12)$, we derive

$$
\left.\begin{array}{l}
\left(L a_{11}+L^{\prime} a_{11}^{\prime}\right) U+\left(L a_{12}-L^{\prime} a_{12}^{\prime}\right) V=0, \\
\left(L a_{12}-L^{\prime} a_{12}^{\prime}\right) U+\left(L a_{22}+L^{\prime} a_{22}^{\prime}\right) V=0 .
\end{array}\right\}
$$

The condition of compatibility of these equations is the characteristic equation for instability

$$
\left(L a_{11}+L^{\prime} a_{11}^{\prime}\right)\left(L a_{22}+L^{\prime} a_{22}^{\prime}\right)-\left(L a_{12}-L^{\prime} a_{12}^{\prime}\right)^{2}=0 .
$$

This equation corresponds to the spontaneous appearance of sinusoidal deformation of the interface. Since the equation is independent of the parameter $l$, all wavelengths are equally unstable. The result is mathematically analogous to the existence of Stoneley waves which propagate at the interface of two elastic solids with a phase velocity independent of the wavelength.

We shall discuss this equation numerically in a particular case of two media of rubber-like elasticity. Consider a uniform finite plane strain defined by an extension ratio $\lambda$ smaller than unity and in the $x$ direction. The initial compressions are

$$
P=\mu_{0}\left(\frac{1}{\lambda^{2}}-\lambda^{2}\right), \quad P^{\prime}=\mu_{0}^{\prime}\left(\frac{1}{\lambda^{2}}-\lambda^{2}\right) .
$$

As shown in earlier papers ( $1961,1963 b)$, the materials remain isotropic under initial stress and the elastic coefficients are

$$
N=Q=\frac{1}{2} \mu_{0}\left(\lambda^{2}+\frac{1}{\lambda^{2}}\right), \quad N^{\prime}=Q^{\prime}=\frac{1}{2} \mu_{0}^{\prime}\left(\lambda^{2}+\frac{1}{\lambda^{2}}\right) .
$$

The value of $\zeta$ is the same for both media,

$$
\zeta=\zeta^{\prime}=\frac{1-\lambda^{4}}{1+\lambda^{4}},
$$

and

$$
m=m^{\prime}=\frac{1}{1+\zeta}, \quad k=k^{\prime}=\sqrt{\frac{1-\zeta}{1+\zeta}} .
$$

Also,

$$
L=\frac{\mu_{0}}{\lambda^{2}}, \quad L^{\prime}=\frac{\mu_{0}^{\prime}}{\lambda^{2}} .
$$

Hence,

$$
\left.\begin{array}{l}
a_{11}=a_{11}^{\prime}=\sqrt{ }\{2(m+k)\}, \\
a_{12}=a_{12}^{\prime}=k-1, \\
a_{22}=a_{22}^{\prime}=k \sqrt{ }\{2(m+k)\} .
\end{array}\right\}
$$

The characteristic equation is

$$
\frac{2 k(m+k)}{(1-k)^{2}}=\left[\frac{\mu_{0}-\mu_{0}^{\prime}}{\mu_{0}+\mu_{0}^{\prime}}\right]^{2} .
$$


If we express $m$ as a function of $k$, this equation is written

$$
k\left(\frac{1+k}{1-k}\right)^{2}=\left(\frac{\mu_{0}-\mu_{0}^{\prime}}{\mu_{0}+\mu_{0}^{\prime}}\right)^{2} .
$$

The case where the material is allowed to expand freely in a direction perpendicular to the $x, y$ plane leads to the same equation. The incremental coefficients for this case were discussed in earlier work (196r). The only difference lies in the value of $\zeta$, which must now be replaced by

$$
\zeta=\left(1-\lambda^{3}\right) /\left(1+\lambda^{3}\right)
$$

Equation (3.10) is a functional relationship between $\zeta$ and the rigidity ratio $\mu_{\mathbf{0}}^{\prime} / \mu_{\mathbf{0}}$. The numerical solution is given in table 1 .

Table 1. Critical value of $\zeta$ for surface instabiutty as a FUNCTION OF THE RIGIDITY RATIO

\begin{tabular}{ll}
$\mu_{0}^{\prime} / \mu_{0}$ & \multicolumn{1}{c}{$\zeta$} \\
0 & 0.83929 \\
0.2 & 0.92407 \\
0.4 & 0.97368 \\
0.6 & 0.99482 \\
0.8 & 0.99972 \\
$1 \cdot 0$ & $1 \cdot 0$
\end{tabular}

The case $\mu_{0}=\mu_{0}^{\prime}$ is that of the homogeneous material. The corresponding value $\zeta=1$ cannot be attained, since it requires $\lambda=0$, i.e. an infinite compression.

The case $\mu_{0}^{\prime}=0$ is that of a half-space with a free surface analyzed earlier.

The characteristic equation $(3 \cdot 10)$ in this case becomes

$$
k^{3}+k^{2}+3 k-1=0 .
$$

Multiplying this equation by $1-k$, we have

or

$$
\begin{aligned}
& 4 k-\left(k^{2}+1\right)^{2}=0, \\
& (1+\zeta)^{2} k-1=0 .
\end{aligned}
$$

In terms of $\zeta$, the rational form of this equation is

$$
\zeta^{3}+2 \zeta^{2}-2=0
$$

whose real root is $\zeta=0 \cdot 83929$. Equations $(3 \cdot 14)$ and $(3 \cdot 15)$ were derived by the writer in the earlier papers.

\section{ReFterenche}

Biot, M. A. I96 I Surface instability of rubber in compression. Air Force Office of Scientific Research, Report T.N. 1771 (to be published in Applied Scientific Research, Ser. A).

Biot, M. A. I963a Proc. Roy. Soc. A, 273, 306.

Biot, M. A. 1963b Proc. Roy. Soc. A, 273, 329. 